\section{CIENCIA Y POLÍTICA EN EL EXILIO REPUBLICANO DE 1939: UN ETHOS CIENTIFICISTA PARA LA LEGITIMIDAD REPUBLICANA}

\author{
José María López Sánchez \\ Departamento de Historia Contemporánea \\ Facultad de Geografía e Historia \\ Universidad Complutense de Madrid \\ jmlopezs@ghis.ucm.es
}

\section{SCIENCE AND POLITICS IN THE 1939 REPUBLICAN EXILE: THE ETHOS OF SCIENTISM FOR REPUBLICAN LEGITIMACY}

Cómo citar este artículo/Citation: López Sánchez, J.M. (2014). "Ciencia y política en el exilio republicano de 1939: un ethos cientificista para la legitimidad republicana". Arbor, 190 (765): a104. doi: http://dx.doi.org/10.3989/arbor.2014.765n1011

Recibido: 22 marzo 2013. Aceptado: 12 noviembre 2013.

RESUMEN: Este artículo trata de analizar la vinculación entre ciencia y política en el exilio republicano de 1939. Esta relación se forjó en torno a la construcción de un ethos cientificista que defendía el ejercicio de una ciencia moderna porque los valores morales y éticos derivados de la misma tenían que validar el programa político liberal y democrático del exilio republicano. De esta forma los científicos exiliados entendieron que su mejor contribución a la resistencia antifranquista pasaba por la elaboración de un discurso opositor basado en las virtudes de la ciencia moderna, antítesis de la cosmovisión científica que poseía el academicismo franquista, anquilosada en una concepción medieval de la ciencia. Las páginas de la revista Ciencia, fundada en 1940, fueron su testimonio.

PALABRAS CLAVE: Exilio español republicano; ciencia; política; resistencia antifranquista.
Copyright: (C) 2014 CSIC. Este es un artículo de acceso abierto distribuido bajo los términos de la licencia Creative Commons Attribution-Non Commercial (by-nc) Spain 3.0.
ABSTRACT: This article seeks to explore the link between science and politics in the Republican exile of 1939. This relationship was forged around the construction of an ethos of scientific thought that defended the pursuit of modern science because the moral and ethical values derived from it had to validate Republican exiles' liberal and democratic political agenda. Thus, exiled scientists considered their best contribution to the anti-Franco resistance was to develop a discourse of opposition based on the virtues of modern science, which was the antithesis of the academic scientific worldview held by Franco's dictatorship, stuck in a medieval conception of science. The pages of the journal Ciencia, founded in 1940, bear witness to it.

KEYWORDS: Spanish Republican exile; science; policy; antiFranco resistance. 
La guerra civil frenó en seco la consolidación de un sistema científico en España, cuyas bases se habían sentado a lo largo del primer tercio del siglo XX gracias a la labor impulsada desde la Junta para Ampliación de Estudios, pues "a pesar de los altibajos, el resultado fue la génesis de una comunidad científica joven, dinámica, bien articulada, con participación activa en el contexto internacional, [...]. Pero ese colectivo sufrió una profunda crisis entre 1936 y 1939, como consecuencia del golpe militar y del desastre provocado por la Guerra Civil y la ulterior represión franquista" (Barona, 2010, 10). Las raíces ideológicas y culturales de la dictadura del general Franco constituyeron un retroceso de alcance histórico para el todavía frágil entramado científico español, mientras el exilio representó la sangría de una parte sustancial del capital humano de la cultura española, incluido el componente científico, dando lugar a una descapitalización que tardó decenios en ser solventada. La depuración emprendida por los vencedores de la guerra civil cercenó bruscamente el proceso modernizador registrado a lo largo del primer tercio del siglo XX. El sistema científico español, que en los años veinte y treinta había emprendido una senda esperanzadora, situándolo por primera vez en la época contemporánea en el panorama científico internacional, fue borrado de un plumazo. Se expulsó a científicos de reconocido prestigio internacional, se destruyeron escuelas y grupos de investigación consolidados, se desmantelaron instituciones científicas y centros de investigación (Otero, 2006).

Se cercenó, en fin, el entramado construido con la $J A E$, se persiguió con saña el espíritu que la alentaba, se arrasó la apuesta por la modernidad que encarnaba, en la que la sociedad española se había embarcado con creciente fuerza y dinamismo desde principios del siglo XX, para entronizar una trasnochada retórica imperial que buscaba sus raíces en el espíritu de la Contrarreforma, como dejó bien claro el preámbulo de la ley sobre Ordenación de la Universidad española de 29 de julio de 1943:

“Cuando adviene la unidad nacional y suena la hora
universal de España, nuestra Universidad, [...] aparece
en la plenitud de su concepto para servir los ideales
de su destino imperial [...] Vivíamos momentos de cri-
sis y de ruina en que si la educación intelectual estaba
desquiciada, había sucumbido también en manos de
la libertad de Cátedra la educación moral y religiosa,
y hasta el amor a la Patria se sentía con ominoso pu-
dor, ahogado por la corriente extranjerizante, laica,
fría, krausista y masónica de la Institución Libre, que
se esforzaba por dominar el ámbito universitario [...] la República lanzó a la Universidad por la pendiente del aniquilamiento y desespañolización [...] La Ley [...] quiere ante todo que la Universidad del Estado sea católica. Todas sus actividades habrán de tener como guía suprema el dogma y la moral cristiana [...] Por otra parte, la Ley [...] exige el fiel servicio de la Universidad a los ideales de la Falange" ${ }^{1}$.

Del lado de la emigración científica e intelectual, la incertidumbre del retorno era el sentimiento que dominaba en 1939, si bien pocos exiliados se resignaron a aceptar que el destierro fuera permanente. Las circunstancias sociales y personales generadas por la depuración de los cuadros docentes de la universidad española y su reconstrucción mediante la provisión de cátedras desataron la protesta de la emigración universitaria. En los años cuarenta el exilio científico e intelectual se sumó a la denuncia de la represión política y social, desde dos esferas complementarias. Por una parte, defendió la actuación educativa de la Segunda República, desveló los atropellos del franquismo contra la universidad, la investigación científica y el conjunto del sistema educativo. El exilio universitario hizo suya la política republicana en materia de instrucción pública porque veían en ella un respaldo ético para reivindicar la imagen liberal y democrática de la Segunda República y apuntalar la crítica contra todos los abusos cometidos por la dictadura en España. En este contexto, la revista Ciencia ofreció, uniéndose al programa de denuncias, una contrarréplica a la política científica y cultural del franquismo a través de la construcción de un ethos cientificista basado en la defensa de la ciencia moderna y los homenajes a las más destacadas figuras de la ciencia en la emigración.

\section{EL DISEÑO DE LA REVISTA CIENCIA}

El exilio de las ciencias naturales en México se agrupó pronto en torno a la emblemática figura de Ignacio Bolívar, que había llegado a México a sus 89 años, y a la práctica científica de su hijo Cándido, condensadas ambas en la fundación de la revista Ciencia. Revista hispano-americana de Ciencias puras y aplicadas. A finales de noviembre de 1939 el proyecto estaba bastante perfilado y Cándido Bolívar, quien había regresado de su primer trabajo de campo en Chiapas, podía explicar a José Cuatrecasas, exiliado en Colombia, los planes merced a los cuales "un grupo de profesores ibero americanos y españoles, con el apoyo de "Editorial Atlante, S.A.", de México, ha emprendido la tarea de publicar una revista científica mensual en lengua española que, al mismo tiempo que recoja los hechos más salientes del movimiento científico ibero americano, sea una fuente de información para todos los 
colegas del Continente"2. Ignacio Bolívar figuraba al frente de una revista nacida en las conversaciones con su hijo Cándido y el círculo de naturalistas refugiados en México.

Hasta su muerte, el 19 de noviembre de 1944, Ignacio Bolívar ejerció la dirección de la revista. Tras su desaparición el Patronato de Ciencia se reunió el 20 de febrero de 1945 y eligió como nuevo director a Blas Cabrera ${ }^{3}$. Sucedía a Bolívar un nombre emblemático de las ciencias físicas en España, cuya carrera estaba íntimamente ligada a la dirección del Laboratorio de Investigaciones Físicas de la JAE y su posterior incorporación al Instituto Nacional de Física y Química, así como al proyecto de renovación científica de la Junta. El 4 de febrero de 1939 una Orden había separado del servicio activo a Blas Cabrera Felipe ${ }^{4}$, catedrático de Electricidad y Magnetismo y director del citado Instituto Nacional de Física y Química. Desde 1936 Cabrera se había instalado en París hasta que en 1942 se trasladó definitivamente a México. Introductor, junto a Enrique Moles, de una concepción moderna de las ciencias físico-químicas, Cabrera era, además de Miguel Antonio Catalán, el físico más importante que había tenido España a lo largo de su historia y uno de los principales divulgadores de la relatividad einsteniana en el país (Otero y López, 2012; Sánchez Ron, 1991, 1993). Junto a Cajal y Bolívar, Cabrera había sido un tenaz impulsor de las modernas ciencias naturales en la JAE, por lo que pertenecía a aquella generación de "mayores" que habían liderado el proyecto de la Junta durante los años "heroicos" y sobre los que se habían formado las escuelas de trabajo y las generaciones de científicos más jóvenes a lo largo de las siguientes dos décadas y media. Su trayectoria y el desprecio que le obsequió el régimen franquista cuando intentó regresar a España lo empujaron al exilio. Aunque menos comprometido políticamente que Ignacio Bolívar, el reconocimiento internacional de Cabrera lo convertían en el candidato idóneo para suceder al entomólogo al frente de la revista.

Muy breve fue el tiempo que Blas Cabrera ejerció como director porque falleció el 1 de agosto de 1945:

\footnotetext{
"Con el fallecimiento del Prof. Cabrera ha experimentado la revista una pérdida sumamente sensible, pues era uno de los mejores y más entusiastas defensores de Ciencia, a la que amparó en todo momento con su gran prestigio, [...]. La figura señera de Don Blas, junto con las de algunos otros científicos muy distinguidos como Ignacio Bolívar, Pío del Río-Hortega, José Sánchez-Covisa, Wenceslao López Albo, Odón de Buen y algunas más, quedará para siempre en la acogedora tierra de América, como mudo y perenne testimonio
}

de la imposibilidad de convivencia de esos espíritus eminentes con el régimen que les imposibilitó la vida en su patria" 5 .

Antonio Madinaveitia tomó la pluma para escribir en diciembre de 1945 una necrológica en Ciencia cargada de referencias a su trayectoria en los laboratorios de la Junta para Ampliación de Estudios y a su personalidad científica, tan relacionada con las principales figuras que habían protagonizado el renacer de la ciencia y la cultura españolas del primer tercio de siglo:

“España va perdiendo en el destierro a los hombres que impulsaron el intenso desarrollo científico que se fue producido allí desde comienzos del siglo hasta la sublevación militar. Ha muerto José Castillejo, murió D. Ignacio Bolívar y ahora Cabrera [...] Deja D. Blas una labor física importante, y además pudo tener la satisfacción de ver reunidos en su laboratorio un grupo de jóvenes investigadores, unos formados por él desde el comienzo, otros que encontraron en su laboratorio el apoyo y la dirección necesarios para sus investigaciones. Este grupo de hombres jóvenes comenzaba a crear la física española, y lo hubiera logrado de no haber sido por la guerra" (Madinaveitia, 1945, 241-242).

El Patronato "acordó el nombramiento de director de la revista, a partir de 1946, del Prof. C. Bolívar y Pieltain" ${ }^{\prime}$, hasta entonces miembro del comité de redacción y principal encargado de la publicación de la revista. La llegada de Cándido Bolívar a la dirección ponía de manifiesto que el exilio había empezado a cobrarse el tributo generacional de figuras egregias de la ciencia española, cuyos nombres estaban intrínsecamente asociados a la etapa inmediatamente anterior a la guerra civil. Hasta la aparición del último número de la revista en diciembre de 1975 Cándido Bolívar ejerció como director de la misma, por lo que podría decirse que Ciencia casi murió con él. No obstante, desde marzo de 1973 la revista había entrado en una nueva etapa que tenía mucho que ver con el hecho de que Cándido Bolívar se encontraba cada vez más enfermo y fue delegando sus responsabilidades al frente de la misma (Pulgarín, Carapeto y Cobos, 2008, 457-489).

Ciencia tuvo una rara cualidad entre las revistas del exilio, su prolongada existencia. Al contrario que la mayor parte de las publicaciones periódicas del destierro republicano, cuya vida se extinguía con mayor o menor rapidez, Ciencia apareció desde 1940 a 1975, aunque fuera de manera irregular. Esto significa que la revista cubrió la casi plena totalidad cronológica del exilio, registrando las diferentes sensibilidades por las que atravesó el exilio científico. Con Ignacio Bolívar 
como referente simbólico hasta su fallecimiento en 1944, quien verdaderamente llevó la administración fue su hijo Cándido Bolívar, junto al que hay que mencionar a un grupo de profesores exiliados que iban a colaborar de manera intensa con aquella publicación. La dirección se distribuyó en tres cuerpos: director, redacción y consejo de redacción. Este último tuvo una amplísima nómina de colaboradores, entre los cuales no faltaron transterrados de gran peso como Federico Bonet, Pedro Bosch Gimpera, Blas Cabrera, José Giral, Gustavo Pittaluga, Miguel Prados Such, José Royo Gómez, Arturo Duperier, Antonio Trías, Faustino Miranda, Gonzalo Rodríguez Lafora, Antonio Madinaveitia, Manuel Márquez, Alejandro Otero, Augusto Pi-Sunyer, José Puche, Pío del Río Hortega, José Cuatrecasas y Enrique Rioja, entre otros muchos. A ellos habría que añadir una extensa terna de profesores hispanoamericanos y de otros países. Por sus páginas pasaron antes o después la mayor parte de los científicos españoles del exilio.

Además de Cándido Bolívar, el corazón de Ciencia lo constituía su redacción, sección que estuvo, hasta 1950, exclusivamente en manos de los universitarios españoles exiliados: Cándido Bolívar, Francisco Giral e Isaac Costero Tudanca gestionaron sus actividades hasta 1944. Desde comienzos de 1945 y hasta 1950 se fueron incorporando en diferentes etapas miembros del exilio científico español, Bibiano Fernández Osorio-Tafall, Honorato de Castro y Federico Bonet, o importantes representantes del mundo científico mexicano, Manuel Sandoval Vallarta, Alfredo Sánchez-Marroquin, Rafael Illescas Frisbie y Antonio García Rojas ${ }^{7}$. A partir de julio de 1952 Ciencia contó con un vicedirector, responsabilidad que recayó en Francisco Giral ${ }^{8}$. Durante los años cincuenta este equipo no experimentó cambio alguno, pero en 1962 José Puche Álvarez sustituyó a Honorato de Castro y en 1965 se unió a la redacción Guillermo Massieu. A partir de 1968 desapareció la figura de vicedirector, aunque Francisco Giral siguió formando parte de la redacción de la revista, a la que se incorporó ese mismo año José Ignacio Bolívar Goyanes. En 1969 dejaban la redacción Rafael Illescas y Antonio García Rojas, a los que relevó Manuel Madrazo Garamendi. A finales de 1972 se incorporó Dionisio Peláez Fernández en calidad de editor. Al año siguiente Ciencia experimentó una considerable remoción de su equipo administrativo con la consolidación de la figura del editor y la creación de un consejo editorial que quedó integrado por Francisco Giral, José Puche y José Ignacio Bolívar Goyanes.
El principal reto al que se enfrentó la administración de la revista fueron las dificultades económicas. Durante los dos primeros años tanto el Comité Técnico de Ayuda a los Republicanos Españoles (CTARE) como la Junta de Auxilio a los Republicanos Españoles (JARE), dos organismos creados para socorrer a los refugiados republicanos, prestaron su ayuda en forma de subvenciones o inscripciones. Esta ayuda económica resultó decisiva durante los primeros meses porque la revista "no reúne el número de suscriptores que sería nece-

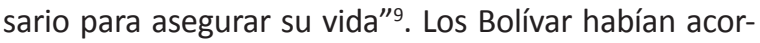
dado con la editorial mexicana Atlante la edición de la revista, pero las perennes dificultades económicas hicieron que Atlante se planteara muy seriamente su continuidad. A lo largo de 1941 y 1942 la precariedad económica era la principal preocupación de Cándido Bolívar, "pues la Editorial se retraía de emplear fondos en una cosa que solo le producía déficit. Se han obtenido algunas subvenciones, que aún no siendo las necesarias para su sostenimiento, han animado a los editores a continuarla"10. Esas otras subvenciones a las que se refería Ignacio Bolívar eran las de algunas empresas y mecenas mexicanos: "como la Compañía Fundidora de Fierro y Acero de Monterrey, el Sr. Santiago Galas y el Banco de México, por gestiones de su Director el Lic. Eduardo Villaseñor. También nos han prestado su auxilio el Gral. Abelardo Rodríguez, ex Presidente de la República; la Secretaría de Marina; D. Arturo Mundet, D. Luis Legorreta, los señores Mendizábal y Cía., de la Cía. Cerillera Mexicana, la Cervecería Moctezuma y la Cía. Hulera Euzkadi, S.A." (Bolívar, 1942, 2).

A la lista de patrocinadores se unieron en $1943 \mathrm{EI}$ Colegio de México, el Fondo de Cultura Económica, los Laboratorios Andrómaco, el Laboratorio Picot y la JARE, que volvió a adquirir un crecido número de suscripciones y cuyo apoyo se mantuvo hasta 1944. Con todo, los últimos tres números del año 1942 tuvieron bastantes dificultades para salir de imprenta, lo que se logró sólo gracias a la ayuda financiera que otorgó la recién creada Comisión Impulsora y Coordinadora de la Investigación Científica en México, cuya presidencia correspondía a Manuel Sandoval Vallarta, un químico mexicano muy sensible a las necesidades del exilio científico republicano. La Comisión Impulsora y Coordinadora de la Investigación Científica en México, que disfrutaba de amplias facultades en temas relativos a la financiación de la política científica, prestó a Ciencia un auxilio imprescindible en el momento en que "hemos pasado por periodos muy difíciles en que parecía indefectible el término de la publicación"11. El sostenimiento económico de la revista terminó por encontrar una solución mer- 
ced a "la idea de crear un Patronato de la Revista Ciencia, que se ocupe de todos los problemas que su publicación origina" (Bolívar, 1943b, 3).

La editorial Atlante se retiró definitivamente en 1946, pero para entonces se había constituido, desde junio de 1943, "un patronato con la misión fundamental de facilitar y regularizar la publicación de nuestra revista. El "Patronato de CIENCIA" estará presidido por el Ing. Evaristo Araiza, y lo integrarán el Lic. Carlos Prieto, como vicepresidente; el Lic. Eduardo Villaseñor, como tesorero; el Prof. Francisco Giral, como secretario, y, como vocales, los Sres. Prof. Blas Cabrera, Don Santiago Galas, Dr. Ignacio González Guzmán, Dr. Manuel Sánchez Sarto, y Prof. C. Bolívar Pieltain"12. Es decir, un órgano colegiado de científicos y mecenas del mundo empresarial que ponía punto final a las incertidumbres económicas. A partir de 1956 la revista consiguió que la Editorial Muñoz se prestara a colaborar en su publicación, contribuyendo a garantizar su permanencia y a conservar su tipografía característica, si bien fue incapaz de mantener una regularidad mensual. Tanto fue así que Cándido Bolívar tuvo que reconocer en 1961 que la revista "es bimestral, y que piensa publicar volúmenes anuales formados por seis números individuales" (Bolívar, 1961, 5). La crisis definitiva llegó entre 1969-72, años en los que el volumen XXVII salió con considerable retraso, producto del cual fue una remoción de su administración en 1973 que no sirvió para salvar la publicación cuando Cándido Bolívar, su verdadera alma mater, cayó enfermo y no pudo seguir al frente con la intensidad de antes.

\section{UNA EMPRESA CIENTÍFICA Y PATRIÓTICA}

El primer número de Ciencia no apareció hasta el 1 de marzo de 1940, pero Cándido Bolívar venía planificando su elaboración desde los últimos meses de 1939, lo que pone de manifiesto la inmediatez con la que algunos integrantes del exilio científico respondieron a los desafíos que parecía plantear la dispersión de sus miembros. Un peligro que los Bolívar querían mitigar a través de un órgano que garantizara un mínimo de contacto personal y profesional. Cándido Bolívar invitó, entre otros, a José Cuatrecasas para que formarse parte del consejo de redacción y, por supuesto, participarse como colaborador asiduo en la revista, cosa que el botánico catalán aceptó con gusto aunque "mi colaboración tal vez no podrá ser por el momento muy copiosa por la falta de materiales de trabajo elaborados y la carencia de biblioteca especializada, defectos que impiden publicar intensamente; no obstante, procuraré exprimirme lo más posible para contribuir a esa empresa científica y patriótica que miro con gran cariño"13. La respuesta de Cuatrecasas confirmaba las dificultades del exilio científico durante estos primeros meses para poder hacer ciencia práctica, investigación científica pura, ya fuese por falta de materiales de trabajo o por las carencias bibliográficas, una consecuencia casi inevitable del desbarajuste vital que supuso el destierro.

Cándido Bolívar le explicaba a Cuatrecasas, en noviembre de 1939, que "La Revista "Ciencia" publicará trabajos originales breves [...]; y al mismo tiempo es nuestra idea presentar un panorama actual de la ciencia moderna en forma de artículos bibliográficos de conjunto (extensión máxima 15 páginas a máquina) ó de puesta al día de un problema actual tanto puramente científico como técnico" ${ }^{14}$, en previsión de que muy pocos compañeros iban a estar en condiciones de poder enviar trabajos originales debido a las condiciones extraordinarias en que vivían. Es más, la revista parecía estar más bien pensada "como foro de debate en el mundo científico americano, más específicamente latinoamericano, y el deseo de participar en la creación de una cultura científica pública" (Puig-Samper, 2001, 107). El objetivo era movilizar al mundo científico, universitario y académico americano, pues a los gestores de la revista les movía: "tan sólo su amor a las Ciencias, que de antiguo vienen profesando, y el anhelo de contribuir al progreso y desarrollo de las mismas en los países hispanoamericanos, animados del deseo de que rivalicen con los más adelantados, y de que la revista pueda llegar a ser un medio de relación entre cuantos se interesan por estos estudios en América" (Bolívar, 1940, 2).

Los Bolívar podían haber optado por postergar la aparición de la revista, pero Cándido la quería en la calle cuanto antes. Esta urgencia tiene mucho que ver con el cariz "científico y patriótico" que tanta simpatía había despertado en Cuatrecasas. Ciencia era una herramienta que permitía la comunicación científica entre la población universitaria disgregada por el vasto continente americano. Pero la revista era algo más, fusionaba la práctica de la ciencia moderna con un proyecto político liberador que la modernidad llenaba de contenido y significado. La revista era una empresa patriótica que sólo casaba con una actividad en teoría tan aséptica como la ciencia si consideramos que Ciencia no era simplemente una publicación sino un tropo (Geertz, 1989, 184), cuyas implicaciones simbólicas difícilmente podían soslayarse. Ciencia condensó la sensibilidad de la emigración científica al convertirse en receptora y sucesora del impulso modernizante y racionalista que había animado el desarrollo de las 
ciencias naturales en España durante las primeras décadas del siglo XX. La idea de hacer "ciencia española" formó parte de una retórica discursiva que había acompañado al academicismo hispánico del primer tercio de siglo. Lo que no parecía tan claro era definir qué se entendía exactamente por "ciencia española". Era un debate que se remontaba hasta mediados del siglo XIX y había sido sostenido, con desigual intensidad, por los tradicionalistas que buscaban armonizar ciencia y fe en un programa común de conocimiento (López, 2011, 1209-1220) y los defensores de una concepción modernizante y racionalista de la ciencia que concebía el progreso como fruto de una investigación científica, libre de dogmatismo religioso, y producto de su aplicación tecnológica (Otero y López, 2012).

Ambos puntos de vista se habían adjudicado para sí los atributos que adornaban a la verdadera "ciencia española". Aunque el paradigma racionalista y modernizante parecía haber conquistado posiciones sólidas tanto en la Universidad como en la Junta para Ampliación de Estudios, la guerra civil y el consiguiente triunfo militar franquista alteraron completamente la situación. El ultramontanismo reaccionario se aseguró el control de los Ministerios de Educación Nacional y Justicia del nuevo régimen, lo que le permitió recuperar terreno en el reparto del poder académico de la posguerra, merced a los procesos de depuración y posterior reconstrucción del mundo universitario, sin olvidar el control ejercido sobre el Consejo Superior de Investigaciones Científicas (Otero, 2006; Claret, 2006). La ciencia en el exilio republicano era la continuadora de los esfuerzos hechos por la JAE y la Universidad durante las décadas anteriores en España. Si la Unión de Profesores Universitarios Españoles en el Extranjero (UPUEE) era, según acertó a definir Francisco Giral, la encarnación de la Universidad española en el exilio (Giral, 1994), Ciencia era la "empresa científica y patriótica" que tanto gustaba a José Cuatrecasas porque ambas constituían símbolos de una tradición de pensamiento emparentado con una cosmovisión político-liberal y científico-racionalista que se resumía en el escorzo lingüístico que Ignacio Bolívar había utilizado en una de sus cartas a Cuatrecasas, "todo por la ciencia y para la ciencia". En definitiva, símbolos y analogías que buscaban excitar las emociones de un exilio intelectual y científico disperso y depresivo, dotándolas de suficiente intensidad para que sus destinatarios, los científicos del exilio, coadyuvaran en un ethos político útil para finiquitar el drama del destierro.

Ciencia tenía muy presente la realidad política y social del destierro republicano, la revista deseaba poner al servicio de México y del resto de repúblicas americanas su talento científico y su práctica investigadora, en agradecimiento por la recepción brindada al exilio, pero sobre todo buscaba sumarse a la contraofensiva cultural que empezaba a gestarse frente a la tosca propaganda imperial franquista. La "Presentación" que abría el primer número de Ciencia recogía esa voluntad por vincularse a los aspectos modernizadores de la ciencia y desarrollar una labor de interés para el mundo hispanoamericano:

\begin{abstract}
"La revista "Ciencia", [...], tiene por finalidad primordial difundir el conocimiento de las Ciencias físiconaturales y exactas y sus múltiples aplicaciones, por considerarlas como una de las principales bases de la cultura pública, para lo que procurará, por todos los medios a su alcance, aumentar el interés hacia su estudio en los países hispano-americanos. De una manera general tratará de tener al lector al corriente de los progresos que aquéllas realicen en todos los órdenes, tanto en su aspecto puramente científico como en sus aplicaciones a la Medicina, a la Agricultura y a la Industria" (Bolívar, 1940, 1).
\end{abstract}

\section{HACER CIENCIA Y HACER POLÍTICA}

Los proyectos científicos del exilio fueron algo más que trabajos de campo y análisis de laboratorio, se transformaron en una forma de hacer política republicana y, por supuesto, la más adecuada para la colectividad de profesores universitarios. Dos de las secciones de la revista, "La Ciencia moderna" y "comunicaciones originales", nunca contuvieron manifiestos políticos, sólo trabajos científicos que, eso sí, respondían a un patrón de compromiso con la modernidad como guía ética para la acción. En "La Ciencia moderna" tenían cabida "artículos redactados por especialistas conocidos, que tratarán problemas científicos de actualidad en su conjunto, poniéndolos por completo al día y ofreciendo, así, [...] la posibilidad de conocer, en algunos minutos de lectura, cuáles son los modernos derroteros en un punto dado de la Ciencia actual" (Bolívar, 1940, 1), una conexión directa con los desarrollos modernos de la Ciencia, escrita siempre con mayúscula. La segunda sección, “Comunicaciones originales”, recibía los trabajos de investigación de los diferentes especialistas, fundamentalmente hispanoamericanos, que tendrían en la revista un foro privilegiado desde el que darse a conocer ellos y sus trabajos.

El Boletín de la UPUEE salía en 1944 al quite de cualquier tipo de reproche acerca del papel desempeñado por la revista Ciencia en este terreno: "Se podría pensar que bajo un nombre científico se encubría actividades políticas contra el régimen franquista. Nada 
más lejos de la realidad. Quien tenga la paciencia de revisar los cuatro volúmenes completos ya publicados y el quinto recién iniciado, no podrá hallar una sola alusión al régimen político en España [...] ni al de ningún país. Como indican título y subtítulo, la revista es exclusivamente científica, y ni siquiera es obra exclusiva de españoles" ${ }^{15}$. No eran, sin embargo, necesarios manifiestos políticos para que Ciencia y la comunidad universitaria expatriada fuesen sensibles a las reivindicaciones políticas del exilio republicano. El mismo editorial del Boletín de la UPUEE subrayaba: "El número elevado de intelectuales españoles que actualmente se hallan fuera de su país por incompatibilidad moral con el régimen que allí domina, y de una manera más concreta la gran cantidad de profesores universitarios de todas clases [...] ausentes de su patria, representa la más viva y constante acusación contra la felonía del régimen franquista y contra su espíritu enemigo de toda manifestación cultural”16. La prohibición decretada contra su distribución en España parecía acreditar que la revista era por sí sola denuncia permanente contra la canalla franquista.

Tanto Ignacio Bolívar como la propia revista Ciencia se convirtieron en referentes simbólicos del exilio científico republicano, catalizadores semióticos que fusionaban la cosmovisión y los valores que la expatriación intelectual ponía al servicio del exilio, su ethos cientificista. A lo largo de los años cuarenta Ciencia fue pilar básico en la construcción de un discurso legitimador de la "ciencia moderna" que permitió a la emigración universitaria contraponer durante los años cuarenta la defensa de los valores modernizadores y democráticos a los abusos de las autoridades franquistas contra los compañeros represaliados. La paralela elaboración de una memoria histórica de la ciencia moderna era un aspecto más de ese discurso. El gobierno Giral, el primer gobierno republicano en el exilio tras la reconstrucción institucional de 1945, representó el momento culminante de la década en la alianza entre el exilio político e intelectual, merced a la cual el primero empleó los argumentos que elaboró el segundo como un instrumento ideológico para justificar las reivindicaciones del republicanismo legitimista. El exilio elaboró un ethos cientificista que reforzaba las exigencias de cambio político en España, apelando a los valores liberales y democráticos compartidos con las potencias vencedoras del fascismo. La ciencia y sus valores éticos hacían más visibles la falta de escrúpulos y la crudeza del terror ejercido por la dictadura franquista.

En ocasiones bastaban trabajos de divulgación o traducciones de obras clásicas para que Ciencia feli- citara a sus autores por su contribución a popularizar la ciencia moderna. En 1940 La Casa de España y el Fondo de Cultura Económica publicaban el libro de José Giral Fermentos, cuyo propósito fundamental "es el de ofrecer al público culto de habla española un resumen moderno y actual de los múltiples problemas científicos y técnicos que se relacionan con los Fermentos. Va dedicado, con preferencia, a Médicos, Farmacéuticos, Biólogos, Químicos e Industriales; y también a estudiantes aventajados de esas profesiones"17. El Fondo y La Casa de España editaron también Las bases fisiológicas de la alimentación de Jaime $\mathrm{Pi}$ Suñer, una línea prioritaria de trabajo seguida por la revista Ciencia a lo largo de las siguientes décadas sobre fisiología y bioquímica de los alimentos en la que destacó no sólo Jaime Pi-Sunyer, también Francisco Giral, Antonio Madinaveitia y otros bioquímicos del exilio científico. Un año más tarde, en 1941, se publicaba en Caracas Principio y término de la Biología de Augusto Pi-Sunyer. De este libro Enrique Rioja decía que era "un sereno y sagaz juicio crítico para valorar y jerarquizar la gran suma de datos, fenómenos, observaciones, experimentos, teorías e hipótesis que constituyen el frondoso cuerpo de doctrina que forma la Biología moderna [...] En 22 capítulos se comentan y analizan los más actuales problemas del campo biológico" (Rioja, 1941, 369).

Mientras la depuración devastaba la universidad española y la investigación científica se retrotraía a parámetros premodernos, las grandes figuras de la ciencia española en el exilio se apresuraban a consolidar su compromiso con la modernidad. Manuel de Rivas Cherif reseñaba las Cuestiones Oftalmológicas de su maestro Manuel Márquez, publicado por El Colegio de México, como obra útil no sólo para oftalmólogos, sino también para fisiólogos, neurólogos e internistas, un libro que repasaba todos los temas de su disciplina y "con decir, que en las páginas de esta obra -que de estar en España le hubiera sido ofrecida por sus discípulos como libro jubilar-, está condensada una gran parte de la labor científica del Prof. Márquez, es suficiente para dar idea del enorme interés que encierra" (Rivas Cherif, 1941, 372). Y no sólo con la modernidad, sino también con la libertad, como Modesto Bargalló reclamaba a raíz de las discusiones abiertas en la comunidad internacional de químicos sobre el uso de la nomenclatura:

"Los investigadores no pueden ni deben someterse espontáneamente a quienes coarten la elevada y noble labor que desarrollan en un campo exclusivamente científico. Sería negar el alto significado de la Ciencia, que junto con los demás valores espirituales 
ha de presidir toda actividad humana. Los que poseen el cetro de la investigación científica no deben rehuir su gran responsabilidad. No olviden que se comienza por la censura de la palabra escrita; que se acalla después a la palabra hablada; y si se dispone de poder para ellos, se termina con aherrojar no sólo al espíritu sino al cuerpo" (Bargalló, 1952, 50).

Otras veces la emigración presumía de contar entre sus filas con los herederos más importantes de la tradición científica en España. Ignacio Bolívar o Blas Cabrera eran la encarnación de ese espíritu, pero otros nombres nutrían sus filas. En abril de 1942 Ciencia se sumó al banquete homenaje que la UPUEE había organizado el 14 de marzo con motivo de que Manuel Márquez había cumplido la edad de setenta años. La revista publicó un retrato del homenajeado, un "oftalmólogo de reputación mundial y figura preeminente del Profesorado español, que hoy continúa en México su labor docente y cultural"18. A finales de 1946, "se celebró un acto en honor del eminente oftalmólogo español Dr. Márquez con ocasión del 50 aniversario de su ejercicio profesional. El homenaje fue organizado conjuntamente por el Ateneo Ramón y Cajal, la Unión de Profesores Universitarios Españoles en el Extranjero y la Agrupación de Universitarios Españoles, [...]. Presidió el acto el Ministro de Instrucción Pública del Gobierno Republicano Español en México, Prof. Miguel Santaló"19.

En aquella mesa se sentaron autoridades del mundo científico e intelectual mexicano y español expatriado como Ignacio Chávez, Ignacio González Guzmán, Rafael Altamira, Juan Negrín, Manuel de Rivas Cherif, José Puche, U. Barnés, José Bejarano, Joaquín Xirau, Antonio Madinaveitia y el grupo de naturalistas formado por Federico Bonet, Dionisio Peláez, Bibiano F. Osorio-Tafall y Cándido Bolívar. Entre los asistentes tomó la palabra Rivas Cherif, discípulo y colaborador de Márquez, para enumerar los méritos profesionales de su maestro. Todo ello hacía de Manuel Márquez, en palabras de Rafael Altamira, un "prototipo del universitario español, y continuador de los valores que, como Santiago Ramón y Cajal, del que fue discípulo distinguido, mantuvieron elevado el pabellón universitario español en el mundo" y, según el ministro Santaló, un "ciudadano ejemplar, que ha sabido en todo momento hacer patente su firme voluntad de servir a España"20. Antes de su fallecimiento, Manuel Márquez fue todavía homenajeado en abril 1957 con motivo de su ochenta y cinco aniversario por el Ateneo Español de México ${ }^{21}$.

Como Ignacio Bolívar o Blas Cabrera, Manuel Márquez constituía un símbolo de los valores que habían de informar al republicanismo exiliado. Esta carga simbólica se repetiría en el banquete homenaje a Cándido Bolívar al ser nombrado Presidente de la Sociedad Mexicana de Historia Natural. Organizado por la UPUEE, el Ateneo Ramón y Cajal y la Agrupación de Universitarios Españoles, el acto contó con la acostumbrada asistencia de destacados exponentes de la intelectualidad mexicana y española: "el Dr. Manuel Márquez, quien se refirió a la actividad científica, en tierras americanas de los profesores españoles y situó el acto bajo la gloriosa memoria del maestro de todos Don Ignacio Bolívar [...]. Este acto que ha sido de los más importantes que los universitarios españoles han celebrado en México estuvo presidido por los homenajeados, [...], y los Sres. Luis Nicolau d'Olwer, Embajador de la República Española y Dr. José Giral, expresidente del Consejo de Ministros de España"22. Estas reuniones reafirmaban la continuidad de los homenajeados con una tradición intelectual vinculada al liberalismo político y la modernidad científica, pero se utilizaron también para celebrar el nombramiento de un científico o intelectual exiliado para un cargo de responsabilidad en alguna institución internacional. Esta clase de éxitos reforzaban los argumentos del legitimismo republicano empeñado en demostrar que tenía la capacidad de conducir un cambio político tranquilo en España. En 1948 la UPUEE, la Delegación General de la Fundación Ramón Llull y la Asociación de Intelectuales Españoles en México rindieron homenaje a Pedro Bosch-Gimpera al ser nombrado director de la Sección de Humanidades y Filosofía de la UNESCO. Asistió una nutrida representación política del gobierno republicano en el exilio: el embajador en México, Luis Nicolau d'Olwer, el antiguo embajador Gordón Ordás y José Giral23.

Junto a los homenajes y reseñas bibliográficas, no tardó en aparecer un tercer instrumento en favor de la ofensiva cultural de la emigración, la memoria del exilio. El 20 de julio de 1944 moría José SánchezCovisa y Ciencia abría por vez primera sus páginas a una necrológica que hacía hincapié en la vinculación de ciencia, política y prestigio internacional en un profesor que

"aparte de su labor docente, [...] desempeñó cargos de tipo académico o cultural como el de decano de la Facultad, que ocupó hasta 1934. [...] Independientemente de su labor científica y docente, al proclamarse la 2a República Española, sintió, como tantos otros universitarios, la necesidad de intervenir en la vida pública del país, siendo elegido Diputado republicano por la provincia de Cuenca en las Cortes Constituyentes, que lo nombraron su Secretario. Era, además, el 
Dr. Sánchez-Covisa miembro de gran número de organizaciones internacionales, habiendo traspuesto su nombre y su personalidad las fronteras de España, dando gloria y prestigio a su patria" (Sánchez-Covisa, 1945, 291).

Unos meses más tarde fallecía Ignacio Bolívar, cuya pérdida generó un torrente de homenajes, y el 29 de diciembre de 1944 moría el psiquiatra Wenceslao López Albo, otro ejemplo de profesional comprometido con la modernidad científica: “En España fue el primer Director y organizador de la Casa de Salud e Instituto Médico para post-graduados de Valdecilla, en Santander (1928-1932), hospital modernísimo [...] En la Casa de Salud Valdecilla vertió el Dr. López Albo los frutos de su vasta experiencia en materia de organización de hospitales, acrecentada por una información muy amplia de los últimos progresos, obtenida merced a la visita de los principales centros científicos de Europa y Estados Unidos", pero además fue ejemplo de moderación y gestión política ya que "durante la guerra española sirvió con todo celo y entusiasmo a la causa legítima de la República, contribuyendo desde los primeros momentos a la eficiente labor de la Sanidad Militar" (Rodríguez, 1945, 130).

Una de las glorias indiscutibles de la ciencia biomédica española, Pío del Río-Hortega, había fallecido el primero de junio de 1945 en Buenos Aires. Quien fuera discípulo de Achúcarro (López, 2012, 547-566) y, por la naturaleza de sus descubrimientos, el más notable continuador de la obra científica de Cajal, fue recordado por su discípulo Isaac Costero Tudanca, que repasó su brillante trayectoria científica y testimonió en su persona los valores que los científicos e intelectuales exiliados querían transmitir:

"Río-Hortega quiso a su Patria con amor desinteresado de niño, con entrega incondicional de amante [...]. Allí dirigió durante muchos años el Laboratorio de Histología Normal y Patológica que la Junta para Ampliación de Estudios e Investigaciones Científicas le confió en la Residencia de Estudiantes, al mismo tiempo que se ocupaba de la dirección del Instituto Nacional de Oncología. Por sus discípulos y por su Patria recorrió las más famosas universidades del mundo [...].Por su desgraciada Patria renunció a puestos brillantes y bien remunerados que le ofrecieron cuando salió de ella" (Costero, 1945, 197).

La fe en el progreso científico y en los valores asociados al mismo era la línea intelectual más sólida que los científicos exiliados podían presentar a la lucha antifranquista. Hacer ciencia equivalía a hacer política y, en este sentido, Ciencia sí era una revista profundamente política, sobre todo al subrayar la coinciden- cia de valores entre el exilio científico y la comunidad científica internacional. En plena Segunda Guerra Mundial la empresa británica "Imperial Chemical Industries" comenzó la edición de Endeavour, Revista trimestral designada a registrar el progreso de las ciencias al servicio de la humanidad e Ignacio Bolívar se detenía a evaluar su significado:

“Ciencia da la bienvenida a la edición española de "Endeavour", de la que viene a ser como una hermana, y sus deseos se confunden íntimamente con los expresados en el artículo de presentación, titulado "Nuestro propósito", debido a Lord McGowan. Cuantos trabajamos en la confección de Ciencia sentimos identificado nuestro propio pensamiento con esta afirmación: "La Ciencia no es el producto de una raza sola, ni (aunque en los últimos 150 años han sido los más significativos) de una edad en particular. Todos los países de Europa han tomado parte en la extensión de las fronteras del conocimiento científico; y el Nuevo Mundo ha resultado ser el par del Viejo". El mismo título de "Endeavour" sugiere agradables remembranzas de una pasada era de descubrimientos" (Bolívar, 1943a, 373).

La ciencia española en el exilio no cejó en su empeño por demostrar que pertenecían a la misma comunidad responsable de ese ensanchamiento de las fronteras del conocimiento. Francisco Giral era tanto o más directo al comentar una conferencia impartida por Karl Taylor Compton, eminente físico norteamericano, presidente del Instituto Técnológico de Massachusetts, que iba a desempeñar un papel fundamental en el desarrollo de la bomba atómica. En una conferencia impartida a comienzos de 1942 Compton

"dedica una mayor atención a la posición y tarea de los científicos norteamericanos en el mundo estadounidense de principios de 1942, con el humo de Pearl Harbor todavía caliente [...]. Después de leído, los científicos que hemos vivido situaciones análogas, quizás de proporciones menores, pero inmensamente más dramáticas y angustiosas, nos quedamos dudando un poco sobre el valor de organizaciones tan perfectas en teoría y de funcionamiento tan complejo como requiere la pretensión de alcanzar lo perfecto. Porque en una situación excepcional y transitoria como lo es toda guerra, al menos para los ciudadanos pacíficos, lo importante es tener resultados útiles rápidamente, con organización o sin ella [...] es muy distinto hacer ciencia de guerra a muchos miles de millas de los frentes de combate que hacer ciencia de guerra cuando las bombas caen a diario en el laboratorio y hasta en el dormitorio del científico" (Giral, 1943, 38).

El reproche crítico de Giral denunciaba lo que él creía cierta ingenuidad del físico norteamericano, 
cuya disertación estaba repleta de datos informativos, pero carecía de "pensamientos de altos vuelos", como ofrecía el esfuerzo de los exiliados.

El exilio era asimismo consciente que el final de la Segunda Guerra Mundial incrementaría exponencialmente el peso de la investigación científica y su complemento, el desarrollo tecnológico. Las naciones que perdían el tren de la ciencia se verían abocadas a desempeñar un papel secundario, el drama de la emigración científica era pensar que la guerra civil había expulsado de España a la elite que tenía que haber liderado esta nueva era científica. En otros países, las ventajas de garantizar la continuidad del progreso científico venían avaladas por organismos como la Smithsonian Institution, que en 1946 cumplía cien años al servicio de la cultura, un hecho que Bibiano Fernández Osorio-Tafall aprovechó para reivindicar lo que el drama del exilio no había permitido en España: "El alma de ella son, sin duda alguna, los hombres que en sus distintos departamentos y oficinas, laboran, al servicio de la ciencia, por el mayor prestigio de la fundación a la que pertenecen. A medida que el tiempo pasa los viejos van cediendo su lugar a los elementos jóvenes, pero hay siempre una continuidad en el tiempo y en los propósitos: el progreso científico [...] CIENCIA se suma jubilosa al homenaje que supone la celebración del Primer Centenario" (Osorio-Tafall, 1946, 249).

El discurso científico-ético del exilio intelectual buscaba sensibilizar la voluntad de las potencias internacionales en lo relativo a la cuestión española. Este propósito vivió un momento especial con la creación de la UNESCO. En enero de 1948 Josephus Needham, director del Departamento de Ciencias Naturales de la misma, escribía en Ciencia una nota en la que presentaba dicha sección y el conjunto de valores que la informaban, compartidos por el destierro español:

\footnotetext{
"Por espacio de cuatro mil años, los grandes conductores espirituales han enseñado la confraternidad esencial entre los hombres, y los deberes de éste hacia sus semejantes. Reforzando esta intuición de la comunidad, se llegó al conocimiento del origen común evolutivo de los seres humanos, presupuesto en la antigua filosofía griega y china, y que culminó con la aceptación del Darwinismo por el mundo moderno. Vivimos en el momento presente, en un mundo tan densamente entretejido por los medios científicos de las comunicaciones y transportes, que lo acaecido a cualquier ser humilde en apartado rincón, puede llegar a afectarnos a todos nosotros. La miseria humana, no menos que la paz mundial, es indivisible; constituyendo el reconocimiento de la unidad fundamental
}

de la humanidad, lo que nos impele a planear la edificación de un nuevo mundo de paz y bienestar social. La Ciencia y la Tecnología por sí solas no pueden hacerlo, pero son absolutamente indispensables, [...] para que ésta se aplique al bienestar social" (Needham, 1948, 302).

En la UNESCO trabajaron activamente, en diferentes etapas, nombres ilustres del exilio intelectual republicano como Pedro Bosch Gimpera, Cándido Bolívar y Augusto Pérez Vitoria ${ }^{24}$, todos cercanos a las actividades de Ciencia.

\section{CONCLUSIONES}

Al estallar la guerra civil, España había asistido en sus décadas precedentes a un notable desarrollo de sus estructuras y capacidades científicas, gracias a la labor emprendida por la Junta para Ampliación de Estudios y su política científica. La Segunda República fue más allá no sólo por su voluntad de sostener los presupuestos de ciencia en un momento de debilidad económica, sino también porque al frente del aparato gubernativo republicano, ya fuese en calidad de jefes de gobierno o de Estado, ministros, diputados y funcionarios de alto rango, operaba con frecuencia una pléyade de intelectuales y profesionales adscritos a muy diferentes ámbitos de la vida científico-cultural del país. Entre los catedráticos universitarios la identificación alcanzó cotas de enorme significación. Aquella República de letras y de ciencias, una República de profesores (Claret, 2006, 516-518), respondía no sólo a las necesidades que en materia educativa e investigadora presentaba el Estado, también se identificó con una tradición política liberal que buscaba regenerar el país desde presupuestos políticos en las antípodas del tradicionalismo político de los círculos más conservadores. Al estallar la guerra civil y superados los primeros meses de disolución del Estado republicano, los más comprometidos se implicaron en la gobernabilidad de la zona leal al gobierno o lo representaron en el extranjero a través de embajadas y misiones culturales.

La reconstitución en 1945 del gobierno republicano en el exilio, en la persona del catedrático de química biológica José Giral, respondía a diversas claves. Para los dirigentes del exilio era un momento histórico clave, tras la derrota del fascismo en la guerra mundial la emigración republicana esperaba que sus demandas fueran atendidas por las grandes potencias encargadas de diseñar el nuevo orden internacional. Los grupos legitimistas querían, por otro lado, conectar con la tradición republicana de los años treinta, quebrada dramáticamente por la guerra civil. Para entonces las elites científicas del exilio tenían hechos sus deberes, 
en torno a Ciencia y a algunas organizaciones como la UPUEE se había ido elaborado un discurso cargado de un fuerte ethos cientificista que contribuyera a reforzar la legitimidad republicana. Para ello se sirvieron, como hemos visto, de homenajes, reseñas y la construcción de un discurso que vinculara al exilio con el ejercicio de una ciencia moderna y sus valores políticos concomitantes. El gobierno Giral era la expresión de una alianza entre ciencia y política dentro de la emigración republicana que aspiraba a liderar la oposición antifranquista desde postulados que superaran las luchas intestinas del republicanismo.

La práctica y el ejercicio de la "ciencia moderna", frente al nacionalcatolicismo franquista, denunciaba por sí sola lo trasnochado del régimen político que regía los destinos de España. En lo político, la ciencia moderna sólo se concebía en un régimen democrático y liberal como el de las democracias vencedoras del fascismo, que eran los destinatarios de este mensaje. Los valores éticos y morales del desarrollo científico y tecnológico eran puestos al servicio de la legitimación republicana, de su capacidad para liderar el cambio político que necesitaba España sin poner en riesgo el estatus quo internacional. De llevarse a cabo la deseada revisión de la "cuestión española" el gobierno Giral quiso ofrecer a los aliados las seguridades de sus elites racionalistas y científicas. Su fracaso supuso el final de un proyecto que había unido a ciencia y política en el exilio. A finales de los años cuarenta, cuando la lógica de la guerra fría garantizó la continuidad del franquismo, científicos e intelectuales del exilio mantuvieron el discurso, pero dejaron la escena política para centrarse en reconstruir los contactos con el interior de España, donde empezó a fraguarse la recuperación de una tradición de pensamiento liberal y moderno alejado de la vacuidad nacionalcatólica.
1 Ley de 29 de julio de 1943 sobre ordenación de la Universidad española, Boletín Oficial del Estado, n.o 212, 31 julio 1943, pp. 7.406-7.408.

2 Carta de Cándido Bolívar a José Cuatrecasas, 28 de noviembre 1939. AHRJB. Fondo José Cuatrecasas. Correspondencia científica, Caja 4. Expediente Div. XV, 2,1,14.

3 "Nuevo director de CIEncIA", Ciencia. Revista hispano-americana de Ciencias puras y aplicadas, VI/2 (27 febrero 1945), p. 66.

4 Archivo General de la Administración (AGA), Educación, expediente personal de Blas Cabrera Felipe, CIDE: 92057, expediente 1. Boletín Oficial del Estado, no48, 17 febrero 1939, p. 932

5 "Nuevo director de CIENCIA", Ciencia. Revista hispano-americana de Ciencias puras y aplicadas, VI/7-9 (20 diciembre 1945), p. 274

6 "Revista Ciencia", Ciencia. Revista hispano-americana de Ciencias puras y aplicadas, VII/1-3 (15 agosto 1946), p. 37.

7 "Consejo editor de "Ciencia", Ciencia. Revista hispano-americana de Ciencias puras y aplicadas, XI/7-9 (15 diciembre 1951), p. 228
8 "Patronato de CIENCIA", Ciencia. Revista hispano-americana de Ciencias puras y aplicadas, XII/3-4 (15 agosto 1952), p. 100.

9 Carta de Ignacio Bolívar a José Cuatrecasas, 1 de agosto 1940. Archivo Histórico Real Jardín Botánico (AHRJB). Fondo José Cuatrecasas. Correspondencia científica, Caja 4. Expediente Div. XV, 2,1,14.

10 Carta de Ignacio Bolívar a José Cuatrecasas, 1 de marzo 1942. AHRJB. Fondo José Cuatrecasas. Correspondencia científica, Caja 4. Expediente Div. XV, 2,1,14.

11 Carta de Ignacio Bolívar a José Cuatrecasas, 7 de enero 1943. AHRJB. Fondo José Cuatrecasas. Correspondencia científica, Caja 4. Expediente Div. XV, 2,1,14.

12 "Patronato de la Revista CIENCIA", Ciencia. Revista hispano-americana de Ciencias puras y aplicadas, IV/8-10 (15 marzo 1944), p. 232

13 Carta de José Cuatrecasas a Cándido Bolívar, 18 de diciembre 1939. AHRJB. Fondo José Cuatrecasas. Correspondencia cientifica, Caja 4. Expediente Div. XV, 2,1,14.

14 Carta de Cándido Bolívar a José Cuatrecasas, 28 de noviembre 1939. AHRJB. Fondo José Cuatrecasas. Correspondencia cientifica, Caja 4. Expediente Div. XV, 2,1,14.
15 "Las publicaciones de los emigrados españoles prohibidas en España. La Revista Ciencia", Boletín informativo de la Unión de Profesores Universitarios Españoles en el Extranjero (sección de México), mayo de 1944, número 10, p. 2.

16 "Las publicaciones de los emigrados españoles prohibidas en España. La Revista Ciencia", Boletín informativo de la Unión de Profesores Universitarios Españoles en el Extranjero (sección de México), mayo de 1944, número 10, p. 1.

17 Extracto de la publicidad hecha en la página final de Ciencia del libro de Giral, Ciencia. Revista hispano-americana de Ciencias puras y aplicadas, I/4 (1 junio 1940).

18 "Dr. Manuel Márquez", Ciencia. Revista hispano-americana de Ciencias puras y aplicadas, III/3-4 (25 abril 1942), pp. 96 y 120.

19 "Homenaje al Dr. Don Manuel Márquez", Ciencia. Revista hispano-americana de Ciencias puras y aplicadas, VII/1-3 (15 agosto 1946), p. 37.

20 "Homenaje al Dr. Don Manuel Márquez", Ciencia. Revista hispano-americana de Ciencias puras y aplicadas, VII/1-3 (15 agosto 1946), p. 38. 
21 "Homenaje al Dr. Márquez en su 85 aniversario", Ciencia. Revista hispanoamericana de Ciencias puras y aplicadas, XVII/1-3 (15 julio 1957), p. 32.

22 "Homenaje al Prof. Bolívar", Ciencia. Revista hispano-americana de Ciencias puras y aplicadas, VIII/3 (10 junio 1947), p. 73.

\section{BIBLIOGRAFÍA}

Bargalló, Modesto (1952): "Nomenclatura química y libertad", Ciencia. Revista hispano-americana de Ciencias puras y aplicadas, XII/1-2, pp. 49-50.

Barona, Josep L. (2010): “Destrucción y diáspora de la una comunidad científica: El exilio republicano español", en Barona, Josep L. (ed.): El exilio científico republicano. Valencia: Universitat de València, Valencia, pp. 9-14.

Bolívar y Pieltain, Cándido (1952): "Centro de Documentación Científica y Técnica de México", Ciencia. Revista hispano-americana de Ciencias puras y aplicadas, XII/3-4, p. 102.

Bolívar, Cándido (1961): "Al lector", Ciencia. Revista hispano-americana de Ciencias puras y aplicadas, $\mathrm{XXI} / 1$, pp. 5-6.

Bolívar, Ignacio (1940): "Presentación", Ciencia. Revista hispano-americana de Ciencias puras y aplicadas, 1/1, pp. 1-2.

Bolívar, Ignacio (1942): "Al lector", Ciencia. Revista hispano-americana de Ciencias puras y aplicadas, III/1, pp. 1-2.

Bolívar, Ignacio (1943a): "Endeavour, Revista trimestral designada a registrar el progreso de las ciencias al servicio de la humanidad. Vol. I. Núm. 1, pp. 1-44, Edit. Imperial Chemical Industries; The Kynoch Press. Londres, 1942 (enero)", Ciencia. Revista hispano-americana de Ciencias puras y aplicadas, III/12, p. 373.

Bolívar, Ignacio (1943b): "Al lector", Ciencia. Revista hispano-america-
23 "Homenaje al Dr. Bosch-Gimpera", Ciencia. Revista hispano-americana de Ciencias puras y aplicadas, IX/1-3 (15 diciembre 1948), p. 47.

24 Augusto Pérez Vitoria dirigió un Centro de Documentación Científica y Técnica de México, auspiciado por la UNESCO y el Gobierno Mexicano, encargado de la publicación de un Boletín mensual

na de Ciencias puras y aplicadas, IV/1, pp. 1-2.

Claret Miranda, Jaume (2006): El atroz desmoche: la destrucción de la Universidad española por el franquismo, 1936-1945. Barcelona: Crítica.

Claret, Jaume (2006): "Cuando las cátedras eran trincheras. La depuración política e ideológica de la universidad española durante el primer franquismo", Hispania Nova. Revista de Historia Contemporánea, 6, pp. 511-529.

Costero, Isaac (1945): “In Memoriam. El Dr. Pío del Río-Hortega", Ciencia. Revista hispano-americana de Ciencias puras y aplicadas, VI/5-6, pp. 193-197.

Geertz, Clifford (1989): La interpretación de las culturas. Barcelona: Gedisa.

Giral, Francisco (1943): "Compton, K., R. W. Trullinger y V. Bush. Los científicos miran al mundo de 1942 (Scientists face the World of 1942). 80 pp. New Brunswick (E.U.), 1942", Ciencia. Revista hispanoamericana de Ciencias puras $y$ aplicadas, IV/1, p. 38.

Giral, Francisco (1994): Ciencia española en el exilio (1939-1989). Barcelona: Anthropos.

López Sánchez, José María (2011): “Sapientia et Doctrina. Ciencias Naturales y poder académico en España durante la Edad de Plata", Arbor. Ciencia, pensamiento y cultura, 187/752, pp. 1209-1220.

López Sánchez, José María (2012): "Una escuela, dos laboratorios: Neurociencias en la Junta para Ampliación de Estudios", Revista de la encargado de proporcionar información bibliográfica muy amplia de los trabajos que integraban los centenares de revistas científicas que el Centro recibía. Véase Bolívar y Pieltain, Cándido (1952): "Centro de Documentación Científica y Técnica de México", Ciencia. Revista hispano-americana de Ciencias puras y aplicadas, XII/3-4, p. 102.

Asociación Española de Neuropsiquiatría, 32/116, pp. 547-566.

Madinaveitia, Antonio (1945): “Don Blas Cabrera Felipe", Ciencia. Revista hispano-americana de Ciencias puras y aplicadas, VI/7-9, pp. 241-242.

Needham, Josephus (1948): "Los científicos y la UNESCO", Ciencia. Revista hispano-americana de Ciencias puras y aplicadas, VIII/10-12, p. 302.

Osorio-Tafall, Bibiano F. (1946): "La Institución Smithsoniana. Cien años al servicio de la cultura", Ciencia. Revista hispano-americana de Ciencias puras y aplicadas, VII/7-8, pp. 247-249.

Otero Carvajal, Luis Enrique (dir.) (2006): La destrucción de la ciencia en España. Depuración universitaria en el franquismo. Madrid: Universidad Complutense.

Otero Carvajal, Luis Enrique y López Sánchez, José María (2012): La lucha por la Modernidad. Las Ciencias Naturales y la Junta para Ampliación de Estudios. Madrid: Residencia de Estudiantes - CSIC.

Puig-Samper Mulero, Miguel Ángel (2001): "La revista Ciencia y las primeras actividades de los científicos españoles en el exilio" en Sánchez Andrés, Agustín y Figueroa Zamudio, Silvia (coords.): De Madrid a México. El exilio español y su impacto sobre el pensamiento, la ciencia y el sistema educativo mexicano. Morelia: Universidad Michoacana de San Nicolás de Hidalgo - Comunidad de Madrid, pp. 95-125.

Pulgarín Guerrero, A.; Carapeto Sierra, C. y Cobos Bueno, J. M. (2008): "Visibilidad de la revista Ciencia. Revis- 
ta Hispano-americana de Ciencias puras y aplicadas", en Velamazán, María Ángeles; Vea, Fernando; Cobos, José y Martín, Cándido (coords.): La Historia de la Ciencias y de la Técnica: Un arma cargada de futuro. Ensayos en homenaje a Mariano Hormigón. Cádiz: Servicio de Publicaciones de la Universidad de Cádiz, pp. 457-489.

Rioja, Enrique (1941): "Pi Suñer, A., Principio y término de la Biología. 399 pp. Biblioteca Venezolana de Cultura. Colección Vargas. Caracas,
1941", Ciencia. Revista hispanoamericana de Ciencias puras y aplicadas, II/10-12, p. 369.

Rivas Cherif, Manuel de (1941): “Márquez, M. Cuestiones Oftalmológicas. El Colegio de México. 370 pp. 203 figs. México, D.F., 1941", Ciencia. Revista hispano-americana de Ciencias puras y aplicadas, II/1012 , p. 372.

Rodríguez Mata, M. (1945): “El Dr. Wencesao López Albo", Ciencia. Revista hispano-americana de Ciencias puras y aplicadas, VI/3, p. 130.
Sánchez Ron, José Manuel (1991): "Los orígenes de la Física española contemporánea: Blas Cabrera y Felipe", Revista Española de Física, 5/2, pp. 64-69.

Sánchez Ron, José Manuel (1993): “El mundo de Blas Cabrera", Boletín de la Institución Libre de Enseñanza, 18, pp. 27-48.

Sánchez-Covisa, Antonio Aparicio (1945): “El Dr. José Sánchez-Covisa, 1881-1944", Ciencia. Revista hispano-americana de Ciencias puras y aplicadas, V/9-12, pp. 291-292. 